\title{
Intractable hypoglycaemia in a patient with advanced carcinoid syndrome successfully treated with hepatic embolization
}

\author{
Angelos Kyriacou, ${ }^{1}$ Was Mansoor, ${ }^{2}$ Jeremy Lawrance, ${ }^{3}$ Peter J. Trainer ${ }^{1}$ \\ ${ }^{1}$ Department of Endocrinology; ${ }^{2}$ Department of Medical Oncology; ${ }^{3}$ Department of Radiology, The Christie NHS Foundation \\ Trust, Manchester Academic Health Science Centre; Manchester; UK
}

\begin{abstract}
A male patient presented at the age of 54 years with metastatic pancreatic neuroendocrine tumour (NET). He was managed with interferon and multiple courses of MIBG therapy which controlled his disease for about seven years. He then developed symptomatic hypoglycaemia which resolved with the introduction of somatostatin analogue treatment and further therapeutic MIBG. However, three years later he was admitted to hospital with severe and intractable hypoglycaemia, which persisted despite treatment with dietary manipulation, diazoxide, longacting octreotide injections, intravenous infusion of dextrose and octreotide and everolimus. Bland hepatic embolization was attempted as a last resort and resulted in prompt and dramatic improvement of his condition with no hypoglycaemia for five months. We recommend that hepatic embolization should be considered in patients with advanced and metastatic NETs accompanied by refractory hypoglycaemia, with the aim of symptomatic relief and palliation, and possibly some survival benefit.
\end{abstract}

Key words: Hepatic embolization, Hypoglycaemia, Neuroendocrine tumour

\section{INTRODUCTION}

Pancreatic neuroendocrine tumours (pNETs) are rare, with an annual incidence estimated at 2-4 per $1,000,000$ population, although given their indolent nature this figure could be an underestimate. ${ }^{1}$ Surgery is considered as first line treatment for pNETs, but it is often not possible at presentation due to extensive and metastatic disease; for patients who cannot have surgery, the therapeutic options include somatostatin analogues (SA), targeted therapies (e.g. sunitinib

\footnotetext{
Address for correspondence:

Angelos Kyriacou, The Christie NHS Foundation Trust; Manchester Academic Health Science Centre; Manchester; UK Counrty United Kingdom Bio statement Consultant in Endocrinology \& Diabetes; E-mail: angelos5@doctors.org.uk Received:16-06-2015, Accepted:18-06-2015
}

and everolimus), chemotherapy and liver-directed therapies. ${ }^{1}$ pNETs are divided into functional and non-functional (tumours that are hormonally-secreting or not, respectively).

Hypoglycaemia in the context of metastatic pNETs is due to either functional transformation of the tumour ${ }^{2}$ or, alternatively, to depletion of hepatic glycogen stores with extensive hepatic metastases. The usual treatment algorithm for hypoglycaemia in such instances includes the use of dietary manipulation, diazoxide and SA. Dietary manipulation involves the intake of small, but multiple, meals with a low glycaemic index. Diazoxide inhibits insulin release; however, its use is limited by frequent adverse effects, especially fluid retention and hirsutism. Octreatide, a synthetic SA, has affinity for sst 2 and sst 5 receptors and can improve 
fasting blood glucose and insulin in both benign and malignant insulinomas for up to three years. ${ }^{3}$ Moreover, the PROMID Study Group has demonstrated in a double-blinded, prospective, randomized-controlled trial that octreotide LAR significantly delays time to tumour progression in patients with metastatic midgut NETs, regardless of functionality-status. ${ }^{4}$ The most favourable outcomes were observed in those patients with low hepatic tumour load and with resection of the primary tumour. ${ }^{4}$

Despite the above "conventional" measures in nonoperable NETs, some patients do not respond, some cannot tolerate the aforementioned therapies due to side effects and others experience relapsing hypoglycaemia, after a period of symptom control. In such cases options include radiolabelled somatostatin analogues, such as Lutetium-177 (with/without capecitabine), mTOR receptor inhibitors, such as everolimus, or chemotherapy, such as streptozotocin5FU or capecitabine-temozolamide. ${ }^{5}$

We therefore present our experience in treating a patient with refractory hypoglycaemia due to a metastatic pancreatic carcinoid neuroendocrine tumour (NET); his condition became unresponsive to any of the 'conventional' hyperglycaemic therapies and, indeed, everolimus; instead, he showed a remarkable response to hepatic embolization.

\section{SUBJECTS AND METHODS}

\section{Clinical case}

A 54-year old man presented in 2002 with abdominal pain, but no symptoms suggestive of carcinoid syndrome or hypoglycaemia. A computed tomography (CT) scan showed a pancreatic mass with retroperitoneal and mediastinal lymph nodes and the presence of multiple liver and bone metastases. His urinary 5 hydroxy-indoleacetic acid (5HIAA) level was raised. A CT guided biopsy of the retroperitoneal mass revealed a well-differentiated NET of the pancreas (MIB-1 score $<2 \%$ ).

Given the burden and spread of disease, surgery was deemed inappropriate. Instead, the patient was commenced on interferon-2 $\alpha$ therapy. A meta-iodobenzylguanidine (MIBG) scan done in 2004 showed increased uptake in the lower abdomen as well as in the known metastatic deposits. Interferon- $2 \alpha$ therapy was discontinued and between March 2005 and October 2009 he received four fractions of iodine-131 MIBG. Abdominal and bone pain plus occasional flushing were well controlled, albeit requiring continued supportive therapy with opioids and zolendronic acid. Radiologically, there was a trend towards progression of liver disease.

In September 2009, he developed symptomatic hypoglycaemia, associated with episodes of loss of consciousness, which was confirmed biochemically. In March 2010, he commenced octreotide LAR 20 mg every four weeks and received a fifth fraction of Iodine-131 MIBG therapy, which resulted in resolution of his hypoglycaemic episodes. Octreotide LAR controlled his hypoglycaemic symptoms until February 2013. At this point, hypoglycaemic symptoms were paralleled by tumour progression and a general deterioration in his condition. Diazoxide $(150 \mathrm{mg}$ daily) was added to octreotide but he continued to have increasingly frequent hypoglycaemic episodes culminating in a severe, intractable hypoglycaemic attack, necessitating hospital admission. Despite continuous intravenous infusions (IVI) of $10 \%$ dextrose and octreotide ( $300 \mu \mathrm{g} / 24 \mathrm{hrs})$ he had persistent hypoglycaemia. The addition of everolimus (10 $\mathrm{mg}$ once daily) was of no benefit.

In view of the large volume of hepatic disease (Figure 1), bland left hepatic artery embolization (Figure 2) with $1.5 \mathrm{mls}$ of Spherical Polyvinyl Alcohol (Embozene ${ }^{\circledR}$ Color-Advanced Microspheres, CeloNova BioSciences, Inc., San Antonio, USA), containing 400 microns, was given and resulted in a dramatic reduction in the frequency and severity of hypoglycaemia within 24 hours.

His octreotide and glucose IVI were discontinued within 72 hours. The patient was discharged home on octreotide LAR $30 \mathrm{mg}$ and everolimus $10 \mathrm{mg}$; the latter was continued for its potential anti-tumour action. He remained asymptomatic with no hypoglycaemic episodes for five months before hypoglycaemia recurred and he died two weeks later.

\section{DISCUSSION}

Our case demonstrates that with disease progression in metastatic pNETs it is often not possible to 


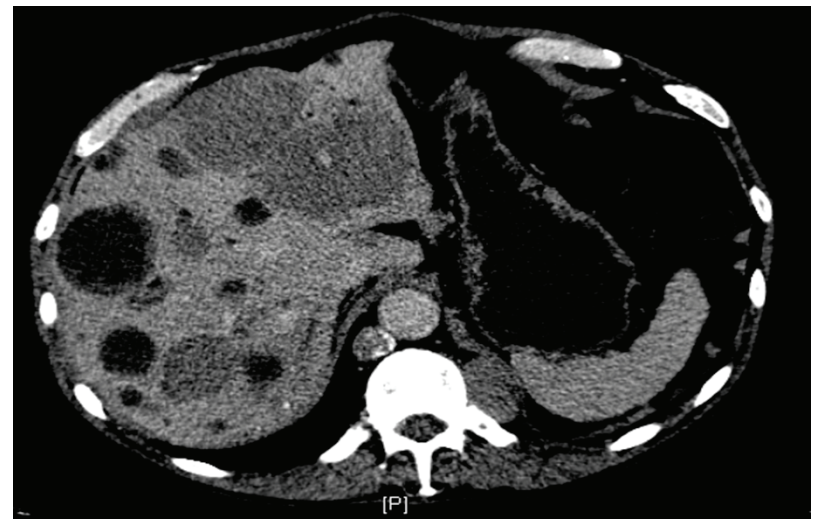

Figure 1. Coronal CT scan of the abdomen performed pre-embolization showing extensive hepatic metastases, some of which are necrotic. A left paraspinal mass is also present on this image. The left hepatic lobe was embolized in preference, given that it contained more tumour bulk and the metastatic lesions were solid rather than cystic and, therefore, more likely hormonally active.

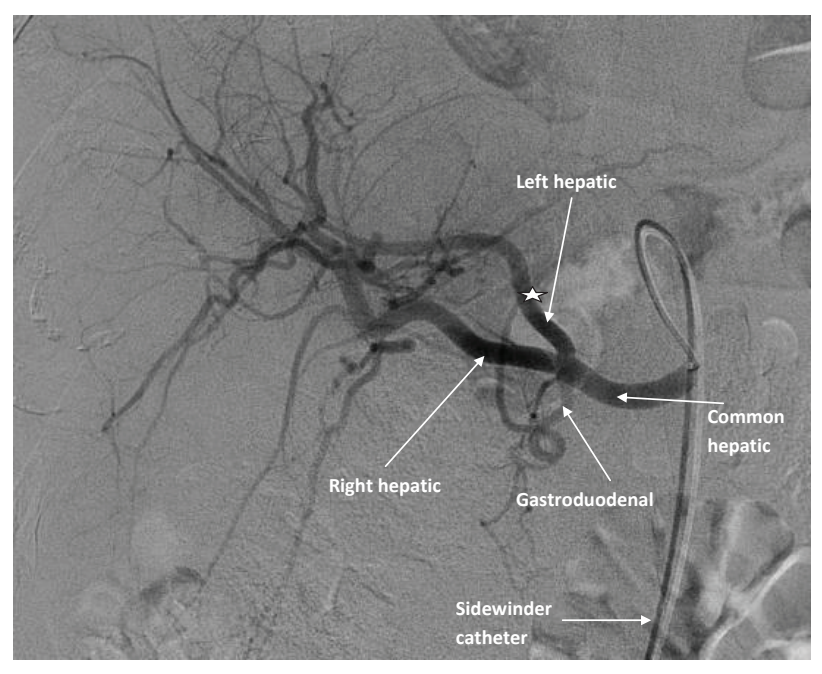

Figure 2. Hepatic artery embolization. Left hepatic arterial angiogram captured during embolization of the left hepatic artery, showing the left, right hepatic and gastroduodenal arteries. Note that the catheter needs to be advanced beyond the gastroduodenal artery to avoid embolization of the pancreas, stomach and duodenum.

* signifies the site of injection of the microspheres (a microcatheter is inserted via a guiding catheter).

achieve symptomatic control with 'traditional' treatment modalities. Newer therapies should be considered, which in the case of symptomatic and refractory hypoglycaemia include everolimus, radiolabelled peptides and hepatic embolization.
The cause of the recurrent hypoglycaemia in our patient is likely to relate to the development of inappropriate insulin co-secretion from his NET (as previously described ${ }^{2}$ ) and/or depletion of his hepatic glycogen stores as the disease evolved, but, unfortunately, insulin and C-peptide were not measured. Hypoglycaemia due to glucagon-like peptide 1 (GLP1) secretion has also been reported in a patient with a strumal ovarian carcinoid syndrome; co-secretion of somatostatin by the same tumour meant that the patient was also suffering with (predominantly fasting) hyperglycaemia. ${ }^{6}$

As Besser et al have reported, the hyperglycaemic "adverse-effect" of everolimus can be utilized in the management of hypoglycaemia secondary to NET. ${ }^{7}$ In addition, everolimus has been shown in a randomized-controlled trial to significantly lengthen progression-free survival among patients with progressive advanced pNETs whilst being well tolerated. ${ }^{8}$ Our patient was not fit for surgery and, given the lack of benefit from everolimus, his frailty (performance score of 3) and personal preference, chemotherapy was not appropriate.

Radiolabelled peptides (RP) can be used to treat recurrent disease refractory to other treatment modalities. Van Schaik et $\mathrm{al}^{9}$ have described resolution of hypoglycaemia with ${ }^{177} \mathrm{Lu}$ octreotate and ${ }^{111} \mathrm{In}$ octreotide in patients with metastatic insulinomas. This treatment modality was not offered, in view of the fact that his lesions did not display any uptake on somatostatin receptor scintigraphy.

Hepatic artery embolization (HAE) can take several forms. We used PVA into the left artery as a means of inducing intra-tumoural ischaemia, alternative forms being chemoembolization (HACE) and selective internal radiotherapy (SIRT). Bland embolization improves symptoms, tumour markers and radiological appearances. ${ }^{10}$ HACE delivers chemotherapy (e.g. with doxorubicin) attached to the embolic agent. It achieves about a twenty-fold higher chemotherapy concentration comparative to systemic chemotherapy with reduced risk of systemic adverse events. ${ }^{10}$ However, there is a dearth of evidence that HACE has a survival benefit compared to bland embolization. Both therapies often require multiple treatment courses; indeed, we were hoping to re-treat our patient if his overall general state improved, but this never materialized. 
Normal hepatic tissue is very radiosensitive, which precludes conventional external beam radiotherapy, but SIRT combines embolization with delivery of tumour localized radiation in the form of microspheres of glass or resin labelled with ${ }^{90} \mathrm{Y}$ (a pure beta emitter) to deliver radiation directly into the hepatic artery, via a percutaneous transcatheter technique. ${ }^{10,11}$ It may offer a biochemical and radiological advantage over other forms of embolization and possibly increases survival, though it requires more focused delivery and is expensive. ${ }^{10}$ SIRT was not offered to our patient because by the time this technology was available, our patient was very frail, but also for reasons of patient preference and lack of proven efficacy in the context of NETs.

More recently, a Japanese team have also documented the utility of hepatic embolization in a 45-year old woman with newly diagnosed and aggressive (Ki67 labelling index of 27\%) metastatic malignant insulinoma with hypoglycaemia refractory to dietary manipulation, dextrose IVI and diazoxide. ${ }^{12}$ Unlike our case, she was not given everolimus nor any chemotherapy (at any stage pre-embolization) and, although octreotide was attempted, it was promptly discontinued because of an allergic reaction. ${ }^{12}$ Other notable differences from our case were the two courses of embolization (one on either hepatic lobe) that were applied ten days apart; ${ }^{12}$ we performed half, rather than whole, liver embolization as the latter carries a significant risk of liver failure. Similarly to our case, there was rapid response to hepatic embolization (within 24 hours), albeit more short-lived (hypoglycaemia recurred after 1.5 months). ${ }^{12}$

This case illustrates the value of hepatic embolization in relieving hypoglycaemia, therefore allowing this patient to live his last five months at home, free from any hypoglycaemic attacks. Hepatic embolization is a cost-effective treatment that should be considered for symptomatic relief in patients with inoperable hepatic metastases causing refractory hypoglycaemia or other hormone-related symptoms.

\section{DECLARATION OF INTEREST}

All authors declare that they do not have any conflict of interest.

\section{FUNDING}

No funding was required for this study.

\section{CONSENT}

Written consent was obtained from the patient's next of kin.

\section{REFERENCES}

1. Valle JW, Eatock M, Clueit B, Gabriel Z, Ferdinand R, Mitchell S, 2014 A systematic review of non-surgical treatments for pancreatic neuroendocrine tumours. Cancer Treatm Rev 40: 376-389.

2. Hinchliffe E, Allcock RL, Mansoor W, Myers MA, 2011 A patient with a metastatic gastroenteropancreatic endocrine carcinoma causing hyperinsulinaemic hypoglycaemia and the carcinoid syndrome. Ann Clin Biochem 48: 579-583.

3. Usukura M, Yoneda T, Oda N, et al, 2007 Medical treatment of benign insulinoma using octreotide LAR: a case report. Endocr J 54: 95-101.

4. Rinke A, Muller H, Schade-Brittinger C, et al, 2009 Placebo-controlled, double-blind, prospective, randomized study on the effect of octreotide LAR in the control of tumor growth in patients with metastatic neuroendocrine midgut tumors: a report from the PROMID Study Group. J Clin Oncol 27: 4656-4663.

5. Ong GS, Henley DE, Hurley D, Turner JH, Claringbold PG, Fegan PG, 2010 Therapies for the medical management of persistent hypoglycaemia in two cases of inoperable malignant insulinoma. Eur J Endocrinol 162: 1001-1008.

6. Todd JF, Stanley SA, Roufosse CA, et al, 2003 A tumour that secretes glucagon-like peptide-1 and somatostatin in a patient with reactive hypoglycaemia and diabetes. Lancet 361: 228-230.

7. Thomas NJ, Brooke AM, Besser GM, 2013 Long-term maintenance of normoglycaemia using everolimus in a patient with disseminated insulinoma and severe hypoglycaemia. Clin Endocrinol (Oxf) 78: 799-800.

8. Yao JC, Shah MH, Ito T, et al, 2011 Everolimus for advanced pancreatic neuroendocrine tumors. N Engl J Med 364: 514-523.

9. van Schaik E, van Vliet EI, Feelders RA, et al, 2011 Improved control of severe hypoglycaemia with malignant insulinomas by peptide receptor radionuclide therapy. J Clin Endocrinol Metab 96: 3381-3389.

10. Harring TR, Nguyen NT, Goss JA, O’Mahony CA, 2011 Treatment of liver metastases in patients with neuroendocrine tumors: a comprehensive review. Int J Hepatol 2011: 154541.

11. Lawandowski RJ, Geschwind JF, Liapi E, Salem R, 2011 Transcatheter intraarterial therapies: rationale and overview. Radiology 259: 641-657.

12. Muro S, Nasu J, Harada R, et al, 2014 Prompt resolution of hypoglycaemia by hepatic transarterial embolization for malignant insulinoma with multiple liver metastases. Acta Med Okayama 68: 307-311. 